\title{
Primary adenocarcinoma of the small intestine presenting as superior mesenteric artery syndrome: A case report
}

\author{
KE-KANG SUN ${ }^{1}$, XIAOYANG WU ${ }^{1}$, GANG LIU $^{1}$, HAIXIN QIAN $^{2}$ and XIAOJUN SHEN ${ }^{1,2}$ \\ ${ }^{1}$ Department of Gastrointestinal Surgery, Kunshan First People's Hospital Affiliated to Jiangsu University, Kunshan, \\ Jiangsu 215300; ${ }^{2}$ Department of General Surgery, First Hospital Affiliated to Soochow University, \\ Suzhou, Jiangsu 215006, P.R. China
}

Received November 25, 2014; Accepted December 14, 2015

DOI: 10.3892/ol.2016.4116

\begin{abstract}
Superior mesenteric artery syndrome (SMAS) is an uncommon cause of vomiting and weight loss due to compression of the third part of the duodenum by the superior mesenteric artery. Small bowel adenocarcinoma is an uncommon tumor, which is frequently delayed in diagnosis as its symptoms and signs are non-specific. The present study describes a case of SMAS occurring in a 51-year-old man, caused by intestinal obstruction secondary to a primary adenocarcinoma of the duodenal-jejunal junction. To the best of our knowledge, the present case is the first report of small bowel adenocarcinoma masquerading as SMAS. The present case highlights the importance of considering the possibility of SMAS in patients with upper bowel obstruction caused by intestinal carcinoma.
\end{abstract}

\section{Introduction}

Superior mesenteric artery syndrome (SMAS) has previously been described under various other names, including, duodenal arterial mesenteric compression, duodenal ileus and Wilkie syndrome (1-3). SMAS is caused by compression of the third part of the duodenum by the superior mesenteric artery (SMA), which takes its origin from the abdominal aorta at the level of the first lumbar vertebra and crosses the duodenum (4,5). The exact prevalence of SMAS worldwide remains unclear, however, the rate has been estimated to be $0.013-0.3 \%$, based on barium studies (6). Treatment is initially conservative, which includes the insertion of a nasogastric tube, mobilization of the patient to a prone, left lateral decubitus position, administration of parenteral nutrition, fluid-electrolyte balance correction and positive nitrogen

Correspondence to: Mr. Xiaojun Shen, Department of General Surgery, First Hospital Affiliated to Soochow University, 296 Shizi Street, Suzhou, Jiangsu 215006, P.R. China

E-mail: fsyx7skk@163.com

Key words: superior mesenteric artery syndrome, small bowel adenocarcinoma balance to increase body weight and restore the retroperitoneal fat tissue (7). In cases where conservative treatment has failed, surgery including Treitz ligament division, gastrojejunostomy, subtotal gastrectomy and Billroth II gastrojejunostomy and duodenojejunostomy may be performed to avoid the risk of duodenal atony and massive dilatation. Numerous predisposing conditions for SMAS, including malignancies, burns, prolonged bed rest, anorexia nervosa, malabsorption, anatomical anomalies and surgical complications, have been identified to have possible impacts on the angle between the SMA and the abdominal aorta (7).

Primary small bowel adenocarcinoma is an uncommon tumor, with non-specific symptoms that may cause a delay in diagnosis and, consequently, a negative outcome (8-11). The duodenum is most frequently involved, followed by the jejunum (12). Small bowel adenocarcinomas are rare, accounting for $<2 \%$ of all tumors of the gastrointestinal tract and $\leq 40 \%$ of all small bowel malignancies in the USA (13). Furthermore, the annual incidence is 1.2-6.5 cases per 1 million individuals. The main treatment for small bowel adenocarcinoma is radical surgical resection (14). The ability to completely resect tumors is one of the most important prognostic factors for survival, and adjuvant chemotherapy is required (15). Small bowel adenocarcinoma exhibits a poor prognosis at all stages of disease, with a 5-year overall survival rate of $14-33 \%$ (16). A considerable number of patients with small bowel carcinoma are diagnosed due to upper small bowel obstruction (12). The present study reports a case of a primary adenocarcinoma of the small intestine causing SMAS. The aim of this report was to highlight that SMA syndrome must be considered a symptom, rather than a disease; therefore, determining the cause of SMA syndrome is important.

\section{Case report}

In August 2014, a 51-year-old man was admitted to the Department of the Gastroenterology, Kunshan First People's Hospital Affiliated to Jiangsu University (Kunshan, China) with symptoms of anorexia, vomiting and epigastric abdominal pain lasting for two weeks. During this two-week period, the patient's body weight had reduced by $8 \mathrm{~kg}$. The patient's past medical history included an endoscopic resection of 
A

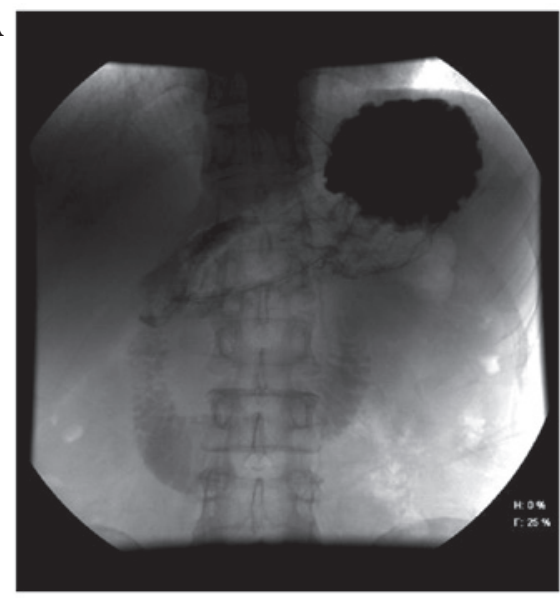

B

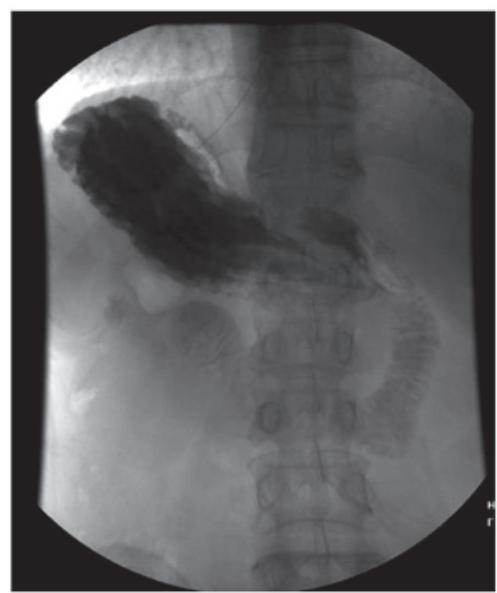

Figure 1. Diatrizoate revealed dilation of the proximal duodenum: (A) Supine position and (B) prone position.

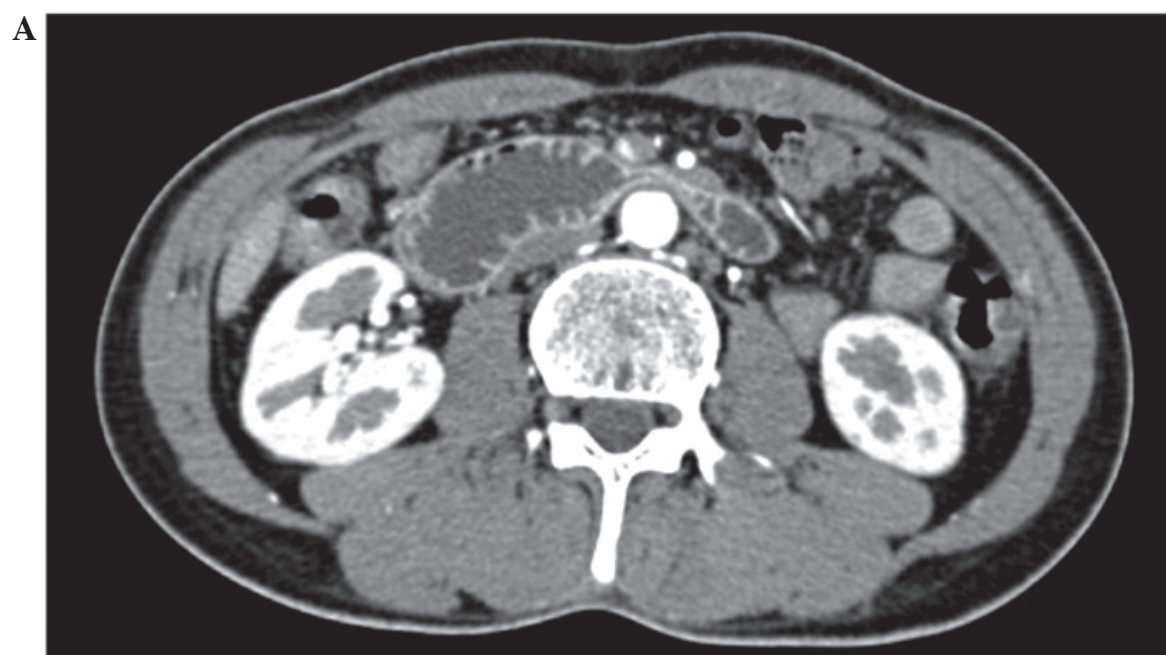

B

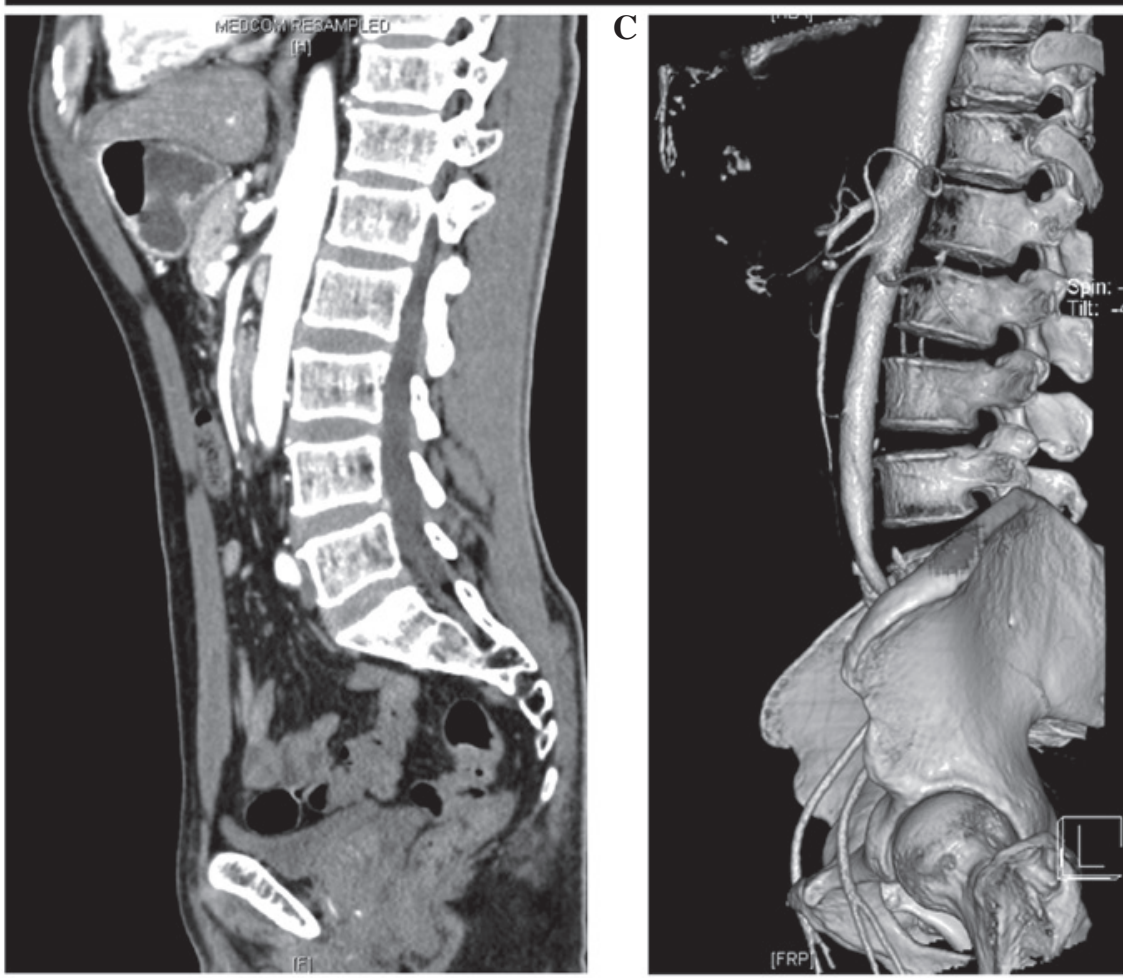

Figure 2. Abdominal computed tomography scans. (A) Abdominal computed tomography revealed distension of the duodenal bulb due to compression of the third part of the duodenum. (B) The distance between the abdominal aorta and superior mesenteric artery was $\sim 8 \mathrm{~mm}$. (C) The angle between the abdominal aorta and superior mesenteric artery was $\sim 20^{\circ}$. 


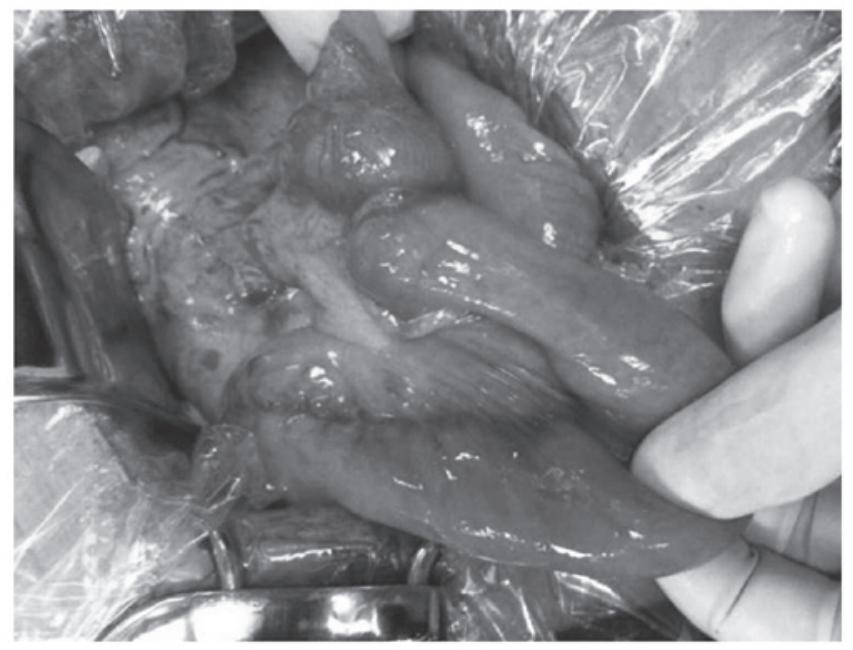

Figure 3. Operative findings revealed a type 2 tumor extension with serosal infiltration at the duodenal-jejunal junction.

colon polyps one month earlier. His medical history and physical examination were suggestive of an upper small bowel obstruction, with symptoms of recurrent bilious vomiting, abdominal pain and upper abdominal distension. The initial blood and urine examinations were within normal ranges. A digital gastrointestinal X-ray machine (PLD7600; Philips, Amsterdam, The Netherlands) was used to perform diatrizoate angiography; diatrizoate (Lunan Pharmaceutical Group Co., Ltd., Shandong, China) revealed dilation of the proximal duodenum, and stenosis of its third part in the supine position (Fig. 1A). In the prone position, the contrast medium passed through the obstructed part of the distal side (Fig. 1B). Contrast-enhanced abdominal computed tomography (CT) scanning (SOMATOM Sensation Cardiac; Siemens AG, Munich, Germany) demonstrated compression of the duodenum between the aorta and SMA, as well as a distended duodenal bulb due to compression of the third portion of the duodenum (Fig. 2A), an aortomesenteric distance of $8 \mathrm{~mm}$ and a reduction of the aortomesenteric angle to $\sim 20^{\circ}$ (Fig. $2 \mathrm{~B}$ and C). Based on the history of symptoms, clinical appearance, the diatrizoate and CT results, SMAS was suspected. A nasogastric tube was inserted and the patient was treated with proton pump inhibitors [Pantoprazole Sodium for Injection (40 mg, daily); Yangtze River Pharmaceutical Group Co., Ltd., Jiangsu, China] and total parenteral nutritional was commenced; however, the symptoms did not improve, and $>800 \mathrm{ml}$ of bilious gastric fluid was drained from the patient each day.

Following two weeks of treatment, it was concluded that conservative measures had failed, and the patient was transferred to the Department of Gastrointestinal Surgery, Kunshan First People's Hospital Affiliated to Jiangsu University, for additional treatment. A laparotomy was performed. During surgery, a duodenal obstruction was confirmed exactly at the point of crossing by the SMA. However, macroscopic examination revealed a type 2 tumor extension with serosal infiltration at the duodenal-jejunal junction, which was causing complete bowel obstruction (Fig. 3). Laparotomy did not reveal direct invasion into the pancreas, peritoneal dissemination or distant metastasis. The ligament of Treitz was separated from the duodenum. Rapid histological diagnosis during surgery revealed moderately-differentiated adenocarcinoma. Partial resection of the duodenum and jejunum, accompanied by lymph node dissection along the superior mesenteric artery, was performed. The inferior pancreaticoduodenal artery and 1 st jejunal artery were ligated for lymphadenectomy. End to end anastomosis was performed between the duodenum and jejunum.

For histological analysis, tissue sections (4-mm) were incubated with $4 \%$ paraformaldehyde (Generay Biotech Co., Ltd., Shanghai, China) for $15 \mathrm{~min}$ at room temperature and washed twice with Tris-buffered saline (TBS) $/ 0.1 \%$ saponin (Generay Biotech Co., Ltd.) for $4 \mathrm{~min}$ each. The sections were then incubated with TBS $/ 0.3 \% \mathrm{H}_{2} \mathrm{O}_{2} / 0.1 \%$ Saponin and $0.02 \% \mathrm{NaN}_{3}$ (Generay Biotech Co., Ltd.) for 30 min to block endogenous peroxidase activity. Next, the sections were washed three times with TBS/saponin for 3 min each then incubated with goat serum [dilution, 1:100; Hangzhou MultiSciences (Lianke) Biotech Co., Ltd., Hangzhou, China] in TBS/saponin for 20 min to block non-specific binding sites. The slides were incubated with an appropriate antibody for immunoblotting, including carcinoembryonic antigen (clone E-4; mouse antihuman monoclonal; catalog no. sc-48374), cytokeratin 20 (clone G-20; goat anti-human polyclonal; catalog no. sc-17113), cytokeratin 7 (clone N-20; goat anti-human polyclonal; catalog no. sc-17116) and cytokeratin 19 (clone M-17; goat anti-human polyclonal; catalog no. sc-33111) (dilution, 1:500; Santa Cruz Biotechnology, Inc., Dallas, TX, USA) overnight at $4^{\circ} \mathrm{C}$. The slides were then washed four times with TBS/ saponin followed by incubation with biotinylated secondary antibody, horseradish peroxidase-labeled goat anti-mouse (catalog no. sc-2005) and goat anti-human IgG (catalog no. sc-2457) (dilution, 1:1,000; Santa Cruz Biotechnology, Inc.) for $30 \mathrm{~min}$. Avidin-biotin-peroxidase reagents [Hangzhou MultiSciences (Lianke) Biotech Co., Ltd.] were then added, and the resulting peroxidase activity was revealed following incubation with $0.5 \mathrm{mg} / \mathrm{ml}$ horseradish peroxidase substrate solution [Hangzhou MultiSciences (Lianke) Biotech Co., Ltd.]. The slides were then washed four times in TBS. Tissues were then stained with hematoxylin and eosin and analyzed using an optical microscope (DSX100; Olympus Corporation, Tokyo, Japan).The histological report determined a diagnosis of moderately-differentiated adenocarcinoma, partially composed of poorly-differentiated cells that were perforating the visceral peritoneum, according to the tumor-node-metastasis classification of malignant tumors (American Joint Committee on Cancer) (17). Additionally, 5/18 lymph nodes demonstrated microscopic metastasis. The definitive diagnosis was primary adenocarcinoma of the duodenal-jejunal junction, T4N1M0, stage III (17). The postoperative course was uneventful and the patient was discharged on postoperative day 12 .

\section{Discussion}

SMAS is an uncommon type of upper intestinal obstruction (18). The pathophysiological process of this syndrome, resulting in a decrease in aortomesenteric angle, is commonly regarded as being due to a decrease in retroperitoneal fat following acute weight loss (19). There are a number of known 
aetiologies for SMAS, including malignancies and malabsorption syndromes. Diagnosis of SMAS is dependent on the barium meal findings of duodenal dilation, retention of barium within the duodenum and characteristic vertical linear extrinsic pressure in the third part of the duodenum (19). Previously, angiographic measurement of the aortomesenteric angle was considered the gold standard of diagnosis; an aortomesenteric angle of $<22-25^{\circ}$ and a distance of $<8 \mathrm{~mm}$ were observed to correlate well with SMAS (20). However, due to the invasive nature of angiography, CT scanning or upper gastrointestinal series are now more commonly used for the diagnosis of SMAS (20).

Primary adenocarcinoma of the small intestine is 40-60 times less frequent compared with colon cancer (21). The diagnosis of small bowel adenocarcinoma is frequently delayed as its symptoms and signs are non-specific. It may develop in any location, but is more frequent in proximal segments, particularly the duodenum and upper jejunum (22).

The current study reported a case of primary adenocarcinoma of the small intestine presenting as SMAS. The patient received conservative therapy for two weeks in the gastroenterology department for the treatment of SMAS; however, the upper gastrointestinal obstructive symptoms demonstrated no significant improvement. Laparotomy revealed complete obstruction of the duodenal-jejunal junction by a primary small intestine adenocarcinoma; how the present patient subsequently developed SMAS is unclear. A plausible explanation may be that the significant weight loss induced by the tumor, as well as a reduction in the angle at which the SMA branched from the aorta, led to compression of the third portion of the duodenum.

In conclusion, the present case highlights that SMAS may be considered as a symptom of a disease, rather than a primary diagnosis. Thus, research investigating the cause of the condition is required. In patients with SMAS, if conservative treatment fails, surgery should then be considered as the next available option.

\section{References}

1. Ylinen P, Kinnunen J and Höckerstedt K: Superior mesenteric artery syndrome. A follow-up study of 16 operated patients. J Clin Gastroenterol 11: 386-391, 1989.

2. Hines JR, Gore RM and Ballantyne GH: Superior mesenteric artery syndrome. Diagnostic criteria and therapeutic approaches. Am J Surg 148: 630-632, 1984.

3. Siddiqui MN, Ahmad T and Jaffary A: Retroperitoneal fungal abscess presenting as superior mesenteric artery syndrome. Postgrad Med J 72: 433-434, 1996.
4. Sinagra E, Montalbano LM, Linea C, Giunta M, Tesè L, La Seta F, Malizia G, Orlando A, Marasà M and D'Amico G: Delayed-onset superior mesenteric artery syndrome presenting as oesophageal peptic stricture. Case Rep Gastroenterol 6: 94-102, 2012.

5. Agrawal $\mathrm{S}$ and Patel H: Superior mesenteric artery syndrome. Surgery 153: 601-602, 2013.

6. Agrawal GA, Johnson PT and Fishman EK: Multidetector CT of superior mesenteric artery syndrome. J Clin Gastroenterol 41: 62-65, 2007.

7. Welsch T, Büchler MW and Kienle P: Recalling superior mesenteric artery syndrome. Dig Surg 24: 149-156, 2007.

8. Halfdanarson TR, McWilliams RR, Donohue JH and Quevedo JF: A single-institution experience with 491 cases of small bowel adenocarcinoma. Am J Surg 199: 797-803, 2010.

9. Ruiz-Tovar J, Martínez-Molina E, Morales V and Sanjuanbenito A: Primary small bowel adenocarcinoma. Cir Esp 85: 354-359, 2009 (In Spanish).

10. Reynolds I, Healy P and Mcnamara DA: Malignant tumours of the small intestine. Surgeon 12: 263-270, 2014.

11. Chaiyasate K, Jain AK, Cheung LY, Jacobs MJ and Mittal VK: Prognostic factors in primary adenocarcinoma of the small intestine: 13-year single institution experience. World J Surg Oncol 6: 12, 2008.

12. Overman MJ: Recent advances in the management of adenocarcinoma of the small intestine. Gastrointest Cancer Res 3: 90-96, 2009.

13. Raghav $\mathrm{K}$ and Overman MJ: Small bowel adenocarcinomas - existing evidence and evolving paradigms.. Nat Rev Clin Oncol 10: 534-544, 2013.

14. Delaunoit T, Neczyporenko F, Limburg PJ and Erlichman C: Small bowel adenocarcinoma: A rare but aggressive disease. Clin Colorectal Cancer 4: 241-248, 2004.

15. Chaaya A and Heller SJ: Introduction to small bowel tumors. Tech Gastrointest Endosc 14: 88-93, 2012.

16. Bilimoria KY, Bentrem DJ, Wayne JD, Ko CY, Bennett CL and Talamonti MS: Small bowel cancer in the United States: Changes in epidemiology, treatment, and survival over the last 20 years. Ann Surg 249: 63-71, 2009

17. Sobin LH, Gospodarowicz MK and Wittekind C (eds): TNM Classification of Malignant Tumours. 7th edition. Wiley Blackwell, pp153-9, 2009.

18. Yakan S, Calıskan C, Kaplan H, Deneclı AG and Coker A: Superior mesenteric artery syndrome: A rare cause of intestinal obstruction. Diagnosis and surgical management. Indian J Surg 75: 106-110, 2013.

19. Ahmed AR and Taylor I: Superior mesenteric artery syndrome. Postgrad Med J 73: 776-778, 1997.

20. Molina Rodríguez JL, Martí Obiol R, López Mozos F and Ortega Serrano J: Superior mesenteric artery syndrome. Cir Esp 90: 53, 2012 (In Spanish).

21. Aparicio T, Zaanan A, Svrcek M, Laurent-Puig P, Carrere N, Manfredi S, Locher C and Afchain P: Small bowel adenocarcinoma: Epidemiology, risk factors, diagnosis and treatment. Dig Liver Dis 46: 97-104, 2014.

22. Lu Y, Fröbom R and Lagergren J: Incidence patterns of small bowel cancer in a population-based study in Sweden: Increase in duodenal adenocarcinoma. Cancer Epidemiol 36: e158-e163, 2012. 